\title{
On Bregman-Type Distances and Their Associated Projection Mappings
}

\author{
Juan Enrique Martínez-Legaz ${ }^{1,2}$ (D) Maryam Tamadoni Jahromi ${ }^{3}$. \\ Eskandar Naraghirad ${ }^{3}$
}

Received: 15 May 2021 / Accepted: 31 October 2021 / Published online: 29 November 2021

(C) The Author(s) 2021

\begin{abstract}
We investigate convergence properties of Bregman distances induced by convex representations of maximally monotone operators. We also introduce and study the projection mappings associated with such distances.
\end{abstract}

Keywords Bregman distance $\cdot$ Fixed point $\cdot$ Maximally monotone operator $\cdot$ Banach space

Mathematics Subject Classification 7H10 $\cdot 37 \mathrm{C} 25$

\section{Introduction and Preliminaries}

This paper is motivated by the recent article [9], which introduces a notion of Bregmantype distance associated with a convex representation of an arbitrary maximally monotone operator in such a way that, when the operator is the gradient of a dif-

Dedicated to Professor Franco Giannessi on the occasion of his 85th birthday.

Communicated by Boris S. Mordukhovich.

$凶 \quad$ Juan Enrique Martínez-Legaz

JuanEnrique.Martinez.Legaz@uab.cat

Maryam Tamadoni Jahromi

M.tamadoni@stu.yu.ac.ir; tamadoni_maryam@yahoo.com

Eskandar Naraghirad

eskandarrad@gmail.com; esnaraghirad@yu.ac.ir

1 Department d'Economia i d'Història Econòmica, Universitat Autònoma de Barcelona, Bellaterra, Spain

2 Barcelona Graduate School of Mathematics (BGSMath), Barcelona, Spain

3 Department of Mathematics, Yasouj University, Yasouj 75918, Iran 
ferentiable strictly convex function $f$, the Bregman-type distance associated with its convex representation coincides with the classical Bregman distance induced by $f$ (see Proposition 4). Bregman-type distances have been further studied more recently in $[7,18]$, the latter paper dealing with their associated farthest Voronoi cells.

The aim of this paper is to investigate the convergence properties of such Bregmantype distances as well as to study their associated projection mappings, which we introduce here.

We recall some definitions and lemmas which will be used in the sequel. We start with the following important lemma, which will play a crucial role in the proof of a fixed point result involving Bregman-type projections we will prove in the last section (see Theorem 17).

Lemma 1 [14, Lemma 4] Let $X$ be a nonempty compact convex set in a Hausdorff topological vector space $Y$. Let $A$ be a closed subset of $X \times X$ having the following properties:

(i) $(x, x) \in$ A for every $x \in X$.

(ii) For any fixed point $y \in X$, the set $\{x \in X:(x, y) \notin A\}$ is a (possibly empty) convex set.

Then, there exists a point $y_{0} \in X$ such that $X \times\left\{y_{0}\right\} \subseteq A$.

Throughout the paper, $E$ will be a Banach space with norm $\|$.$\| and dual space E^{*}$. For any $x \in E$, we denote the value of $x^{*} \in E^{*}$ at $x$ by $\left\langle x, x^{*}\right\rangle$. When $\left\{x_{n}\right\}_{n \in \mathbb{N}}$ is a sequence in $E$, we denote the strong convergence of $\left\{x_{n}\right\}_{n \in \mathbb{N}}$ to $x \in E$ as $n \rightarrow \infty$ by $x_{n} \rightarrow x$, and the weak convergence by $x_{n} \rightarrow x$. We say that a function $h: E \times E^{*} \rightarrow$ $\mathbb{R} \cup\{+\infty\}$ is norm $\times$ weak $*$ lower semicontinuous when every sublevel set of $h$ is closed in the norm $\times$ weak $^{*}$ topology of $E \times E^{*}$, that is, in the product topology of the strong topology of $E$ and the weak* topology of $E^{*}$.

We first recall the notion of convex representation of a maximally monotone operator.

Definition 2 (see [17, p. 27]) Let $S: E \rightrightarrows E^{*}$ be a maximally monotone operator. We say that $h: E \times E^{*} \rightarrow \mathbb{R} \cup\{+\infty\}$ represents $S$ if the following three conditions hold:

(i) $h$ is convex and norm $\times$ weak ${ }^{*}$ lower semicontinuous;

(ii) $h\left(x, x^{*}\right) \geq\left\langle x, x^{*}\right\rangle, \forall\left(x, x^{*}\right) \in E \times E^{*}$;

(iii) $h\left(x, x^{*}\right)=\left\langle x, x^{*}\right\rangle$ if and only if $x^{*} \in S x$. The set of all functions $h$ satisfying these properties will be denoted by $H(S)$.

For a given maximally monotone operator $S: E \rightrightarrows E^{*}$, it is well known (see, e.g., [10, Corollary 4.1]) that $H(S)$ has a smallest element and a biggest one. The biggest element is the norm $\times$ weak $^{*}$ lower semicontinuous convex envelope of $\pi+\delta_{G(S)}$, where $\pi: E \times E^{*} \rightarrow \mathbb{R}$ is defined by

$$
\pi\left(E, E^{*}\right):=\left\langle x, x^{*}\right\rangle,
$$

$G(S)$ denotes the graph of $S$,

$$
G(S):=\left\{\left(x, x^{*}\right) \in E \times E^{*}: x^{*} \in S x\right\},
$$


and $\delta_{G(S)}: E \times E^{*} \rightarrow \mathbb{R} \cup\{+\infty\}$ denotes the indicator function of $G(S)$,

$$
\delta_{G(S)}\left(x, x^{*}\right):=\left\{\begin{array}{c}
0 \text { if }\left(x, x^{*}\right) \in G(S) \\
+\infty \text { otherwise. }
\end{array}\right.
$$

The smallest element is the Fitzpatrick function $F_{S}$ associated with $S$, introduced in [16]:

$$
F_{S}\left(x, x^{*}\right):=\left\langle x, x^{*}\right\rangle-\inf _{\left(y, y^{*}\right) \in S}\left\langle x-y, x^{*}-y^{*}\right\rangle .
$$

For more details on the family $H(S)$, see $[8,10,11,16]$.

We recall that the domain of an operator $S: E \rightrightarrows E^{*}$ is the set

$$
\operatorname{dom}(S):=\{x \in E: S x \neq \emptyset\}
$$

and the domains of $g: E \rightarrow \mathbb{R} \cup\{+\infty\}$ and $h: E \times \operatorname{dom}(S) \rightarrow \mathbb{R} \cup\{+\infty\}$ are the sets

$$
\operatorname{dom}(g):=\{x \in E: g(x)<+\infty\}
$$

and

$$
\operatorname{dom}(h):=\{(x, y) \in E \times \operatorname{dom}(S): h(x, y)<+\infty\},
$$

respectively.

Definition 3 [9] Let $S: E \rightrightarrows E^{*}$ be maximally monotone and single-valued on $\operatorname{dom}(S)$. The Bregman-type distance associated with $h \in H(S)$ is the function $D_{h}$ : $E \times \operatorname{dom}(S) \rightarrow \mathbb{R} \cup\{+\infty\}$ defined by

$$
D_{h}(x, y):=h(x, S y)-\langle x, S y\rangle \text {. }
$$

Let us recall that a function $g: E \rightarrow \mathbb{R} \cup\{+\infty\}$ is said to be strictly convex on a convex set $X \subseteq E$ if

$$
g(\lambda x+(1-\lambda) y)<\lambda g(x)+(1-\lambda) g(y), \forall x, y \in X, x \neq y, \forall \lambda \in(0,1) .
$$

In the case when $X=\operatorname{dom}(g)$, we simply say that $g$ is strictly convex.

We recall the classical definition of Bregman distance. Let $g: E \rightarrow \mathbb{R} \cup\{+\infty\}$ be differentiable on int $\operatorname{dom}(g)$, the interior of $\operatorname{dom}(g)$, and strictly convex. The Bregman distance [13] (see also [6,12]) corresponding to $g$ is the function $D^{g}$ : $\operatorname{dom}(g) \times \operatorname{int} \operatorname{dom}(g) \rightarrow \mathbb{R}$ defined by

$$
D^{g}(x, y)=g(x)-g(y)-\langle x-y, \nabla g(y)\rangle, \quad \forall x, y \in E .
$$


It follows from the convexity of $g$ that $D^{g}(x, y) \geq 0$ for all $(x, y) \in \operatorname{dom}(g) \times$ int $\operatorname{dom}(g)$. However, $D^{g}$ might not be symmetric and might not satisfy the triangle inequality.

We recall that a convex function $g: E \rightarrow \mathbb{R} \cup\{+\infty\}$ is said to be proper if $g \not \equiv+\infty$. Its Fenchel conjugate is the lower semicontinuous proper convex function $g^{*}: E^{*} \rightarrow \mathbb{R} \cup\{+\infty\}$, defined by

$$
g^{*}\left(x^{*}\right):=\sup _{x \in E}\left\{\left\langle x, x^{*}\right\rangle-g(x)\right\} .
$$

Proposition 4 [9, Proposition 3.5] Let $g: E \rightarrow \mathbb{R} \cup\{+\infty\}$ proper, continuous, strictly convex, and differentiable on $\operatorname{dom}(g)$. Take $S=\nabla g$ and $h_{g} \in H(S)$ such that

$$
h_{g}\left(x, x^{*}\right):=g(x)+g^{*}\left(x^{*}\right) .
$$

Then, for every $(x, y) \in E \times \operatorname{dom}(g)$, we have

$$
D_{h_{g}}(x, y)=D^{g}(x, y)=g(x)-g(y)-\langle x-y, \nabla g(y)\rangle .
$$

Remark 5 It is worth noticing that the continuity assumption on $g$ implies that $\operatorname{dom}(g)$ is open. Indeed, one has dom $(g)=g^{-1}(\mathbb{R})$, and $\mathbb{R}$ is open in $\mathbb{R} \cup\{+\infty\}$.

The rest of the paper consists of two more sections. In Sect. 2, we establish some convergence properties of Bregman-type distances. In Sect. 3, we introduce and study projections associated with Bregman-type distances; in particular, we give simple sufficient conditions for such mappings to be single-valued, a characterization of the elements of the projection sets, and a fixed point result for compositions of Bregmantype projection mappings with continuous mappings.

\section{Convergence Properties of Bregman-Type Distances}

Let us recall that a function $g: E \rightarrow \mathbb{R} \cup\{+\infty\}$ is said to be coercive if

$$
\lim _{\|x\| \rightarrow \infty} g(x)=+\infty
$$

and supercoercive if it satisfies the stronger condition

$$
\lim _{\|x\| \rightarrow \infty} \frac{g(x)}{\|x\|}=+\infty .
$$

We start with a generalization of [3, Lemma 2.5].

Lemma 6 Let $\left\{x_{n}\right\}_{n \in \mathbb{N}}$ be a sequence in $E$, and assume that $E$ is reflexive, $S: E \rightrightarrows E^{*}$ is maximally monotone and single-valued on $\operatorname{dom}(S)$, and $h \in H(S)$ is weakly continuous in its first variable. If $\left\{x_{n}\right\}_{n \in \mathbb{N}}$ is bounded, then the sequence $\left\{D_{h}\left(x_{n}, y\right)\right\}_{n \in \mathbb{N}}$ is bounded for every $y \in E$ such that $\left(x_{n}, y\right) \in \operatorname{dom}(h)$ and $\operatorname{dom}(h) \cap(E \times\{y\})$ is 
closed. Conversely, if there exists $y \in E$ such that the sequence $\left\{D_{h}\left(x_{n}, y\right)\right\}_{n \in \mathbb{N}}$ is bounded and $h(\cdot, S y)$ is supercoercive, then $\left\{x_{n}\right\}_{n \in \mathbb{N}}$ is bounded.

Proof Let $y \in E$ be such that $\left(x_{n}, y\right) \in \operatorname{dom}(h)$ and $\operatorname{dom}(h) \cap(E \times\{y\})$ is closed, and suppose that $\left\{D_{h}\left(x_{n}, y\right)\right\}_{n \in \mathbb{N}}$ is unbounded. Then, there is a subsequence $\left\{x_{n_{k}}\right\}_{k \in \mathbb{N}}$ which weakly converges to some $x \in E$ and satisfies $D_{h}\left(x_{n_{k}}, y\right) \rightarrow+\infty$. Clearly, $(x, y) \in \operatorname{dom}(h)$, therefore, since $h$ is weakly continuous in its first variable, we have $D_{h}\left(x_{n_{k}}, y\right) \rightarrow D_{h}(x, y) \in \mathbb{R}$, which is a contradiction. Conversely, let $y \in E$ be such that the sequence $\left\{D_{h}\left(x_{n}, y\right)\right\}_{n \in \mathbb{N}}$ is bounded and $h(\cdot, S y)$ is supercoercive, and assume that $\left\{x_{n}\right\}_{n \in \mathbb{N}}$ is unbounded. After passing to a subsequence if necessary, we may assume that $\left\|x_{n}\right\| \rightarrow \infty$; since $h(\cdot, S y)$ is supercoercive, so is $D_{h}(\cdot, y)$, and thus $D_{h}\left(x_{n}, y\right) \rightarrow \infty$, which is absurd.

Definition 7 (see [4, Definition 2.1]) Let $S$ and $h$ be as in Definition 2. A sequence $\left\{x_{n}\right\}_{n \in \mathbb{N}}$ is said to be $D_{h}$-convergent to $x \in \operatorname{dom}(S)$ if $D_{h}\left(x_{n}, x\right) \rightarrow 0$, in symbols $x_{n} \stackrel{D_{h}}{\rightarrow} x$.

Our next result is a generalization of [4, Proposition 2.2], the proof of which we partially follow.

Proposition 8 ( $D_{h}$-convergence) Let $S$ and $h$ be as in Definition 2. For $x \in \operatorname{dom}(S)$ and a sequence $\left\{x_{n}\right\}_{n \in \mathbb{N}}$ in E, the following statements hold:

(i) If $h(\cdot, S x)$ is continuous and $x_{n} \rightarrow x$, then $x_{n} \stackrel{D_{h}}{\rightarrow} x$.

(ii) If $E$ is reflexive, $S$ is strictly monotone, $h(\cdot, S x)$ is supercoercive and $x_{n} \stackrel{D_{h}}{\rightarrow} x$, then $x_{n} \rightarrow x$.

(iii) If $E$ is finite dimensional, $S$ is strictly monotone and $h(\cdot, S x)$ is continuous and supercoercive, then

$$
x_{n} \rightarrow x \Leftrightarrow x_{n} \stackrel{D_{h}}{\rightarrow} x \Leftrightarrow x_{n} \rightarrow x .
$$

Proof (i) If $x_{n} \rightarrow x$, then $\lim _{n \rightarrow \infty} D_{h}\left(x_{n}, x\right)=D_{h}(x, x)=0$, hence $x_{n} \stackrel{D_{h}}{\rightarrow} x$.

(ii) Since $x_{n} \stackrel{D_{h}}{\rightarrow} x$ and $h(\cdot, S x)$ is supercoercive, $\left\{D_{h}\left(x_{n}, x\right)\right\}_{n \in \mathbb{N}}$ is bounded; so, by Lemma 6 , the sequence $\left\{x_{n}\right\}_{n \in \mathbb{N}}$ is bounded and, in view of the reflexivity of $E$, there exists a subsequence $\left\{x_{n_{k}}\right\}_{k \in \mathbb{N}}$ weakly convergent to some $y \in E$. Since $D_{h}$ is lower semicontinuous in its first variable, we have

$$
0=\lim _{n \rightarrow \infty} D_{h}\left(x_{n}, x\right)=\lim _{k \rightarrow \infty} D_{h}\left(x_{n_{k}}, x\right) \geq D_{h}(y, x) \geq 0
$$

so $D_{h}(y, x)=0$, which, by the strict monotonicity of $S$, yields $y=x$. This proves that $x_{n} \rightarrow x$.

Statement (iii) follows from (i) and (ii), since weak and strong convergence are equivalent in finite-dimensional spaces. 


\section{Projections Associated with Bregman-Type Distances}

In this section, we introduce and study the notion of $h$-projection, $h$ being a convex representation of a maximally monotone operator.

Definition 9 Let $S$ and $h$ be as is Definition 2. For a nonempty set $X \subseteq E$, we define the associated $h$-distance function $D_{h}(X, \cdot): E \rightarrow \mathbb{R} \cup\{+\infty\}$ and $h$-projection mapping $P_{X}^{h}: E \rightrightarrows X$ by

$$
D_{h}(X, y):=\inf _{x \in X} D_{h}(x, y)
$$

and

$$
P_{X}^{h}(y):=\left\{x \in X: D_{h}(x, y)=D_{h}(X, y)\right\},
$$

respectively.

The following result establishes sufficient conditions for the nonemptiness of $h$ Bregman projections. It generalizes [1, Proposition 2.1], and its proof is exactly along the same lines.

Proposition 10 (The domain of $P_{X}^{h}$ ) Let $S$ and $h$ be as is Definition 2. If $E$ is reflexive and $y \in E$ is such that $h(\cdot, S y)$ is weakly continuous and $X \subseteq E$ is nonempty and weakly closed, then $P_{X}^{h}(y) \neq \emptyset$ whenever at least one of the following conditions is satisfied:

(i) $X$ is bounded.

(ii) For every unbounded sequence $\left\{x_{n}\right\}_{n \in \mathbb{N}}$ in $X$, there exists a subsequence $\left\{x_{n_{k}}\right\}_{k \in \mathbb{N}}$ such that $\inf _{k} \frac{h\left(x_{n_{k}}, S y\right)}{\left\|x_{n_{k}}\right\|}>\|S y\|$ (this holds, in particular, if $h(\cdot, S y)$ is supercoercive).

Proof Since $h(\cdot, S y)$ is weakly continuous, $D_{h}(\cdot, y)$ is weakly continuous, too. Take a sequence $\left\{x_{n}\right\}_{n \in \mathbb{N}}$ in $X$ such that $\lim _{n \rightarrow \infty} D_{h}\left(x_{n}, x\right)=D_{h}(X, x)$. If condition (i) holds, then the sequence $\left\{x_{n}\right\}_{n \in \mathbb{N}}$ is obviously bounded. If (ii) is satisfied and $\left\{x_{n}\right\}_{n \in \mathbb{N}}$ were unbounded, then we would have

$$
D_{h}\left(x_{n_{k}}, x\right) \geq h\left(x_{n_{k}}, S y\right)-\left\|x_{n_{k}}\right\|\|S y\|=\left\|x_{n_{k}}\right\|\left(\frac{h\left(x_{n_{k}}, S y\right)}{\left\|x_{n_{k}}\right\|}-\|S y\|\right)
$$

by the assumption, we obtain $\lim _{k \rightarrow \infty} D_{h}\left(x_{n_{k}}, x\right)=+\infty$, a contradiction with $\lim _{n \rightarrow \infty} D_{h}\left(x_{n}, x\right)=D_{h}(X, x)$. Therefore $\left\{x_{n}\right\}_{n \in \mathbb{N}}$ is bounded, and hence, since $E$ is reflexive, it has a weakly convergent subsequence $\left\{x_{n_{k}}\right\}_{k \in \mathbb{N}}$. Let $x^{*}$ be the weak limit of $x_{n_{k}}$. Since $X$ is weakly closed, $x^{*} \in X$. This, together with the equalities

$$
D_{h}\left(x^{*}, x\right)=\lim _{k \rightarrow \infty} D_{h}\left(x_{n_{k}}, x\right)=\lim _{n \rightarrow \infty} D_{h}\left(x_{n}, x\right)=D_{h}(X, x),
$$

implies that $x^{*} \in P_{X}^{h}(y)$, and the proof is complete. 
We next give a sufficient condition for $P_{X}^{h}(y)$ to have at most one element.

Proposition 11 (Nonmultiplicity of $P_{X}^{h}$ ) Let $S$ and $h$ be as is Definition 2. If $X \subseteq E$ is convex and $y \in E$ is such that $h(\cdot, S y)$ is strictly convex and $X \subseteq E$ is convex, then $P_{X}^{h}(y)$ has at most one element.

Proof Clearly, the strict convexity of $h(\cdot, S y)$ implies that of $D_{h}(\cdot, y)$, which in turn implies that $D_{h}(\cdot, y)$ has at most one minimizer over $X$.

Combining Propositions 10 and 11, one obtains sufficient conditions for an $h$ projection mapping to be single-valued; in particular, one gets the following simple statement.

Corollary 12 Let $S$ and $h$ be as is Definition 2. If $E$ is reflexive, $X \subseteq E$ is nonempty, convex and closed and, for every $y \in E$, the function $h(\cdot, S y)$ is weakly continuous and strictly convex on $X$, then $P_{X}^{h}$ is single-valued.

The following characterization of the elements of $P_{X}^{h}(y)$ generalizes part of [5, Fact 2.3].

Proposition 13 (Characterization of projections) Let $S$ and $h$ be as is Definition 2. If $X \subseteq E$ is convex and closed, $S: E \rightrightarrows E^{*}$ is single-valued on its domain, $y \in E$, the function $h$ is finite-valued and such that $h(\cdot, S y)$ is subdifferentiable on $P_{X}^{h}(y)$, and $\bar{x} \in X$, then $\bar{x} \in P_{X}^{h}(y)$ if and only if there exists $x^{*} \in \partial h(\cdot, S y)(\bar{x})$ such that

$$
\left\langle x-\bar{x}, S y-x^{*}\right\rangle \leq 0 \quad \forall x \in X .
$$

Proof The statement follows from the equivalence

$$
\bar{x} \in P_{X}^{h}(y) \Longleftrightarrow 0 \in \partial D_{h}(\cdot, y)(\bar{x})+N_{X}(\bar{x}),
$$

with $N_{X}(\bar{x})$ denoting the normal cone to $X$ at $\bar{x}$, that is,

$$
N_{X}(\bar{x}):=\left\{x^{*} \in E^{*}:\left\langle x-\bar{x}, x^{*}\right\rangle \leq 0 \quad \forall x \in X\right\} ;
$$

notice that

$$
\partial D_{h}(\cdot, y)(\bar{x})=\partial h(\cdot, S y)(\bar{x})-S y .
$$

Corollary 14 Let $X, S$, and $h$ be as in Proposition 13. If $y \in E$ is such that $h(\cdot, S y)$ is differentiable on $P_{X}^{h}(y)$, then

$$
\bar{x} \in P_{X}^{h}(y) \Longleftrightarrow\langle x-\bar{x}, S y-\nabla h(\cdot, S y)(\bar{x})\rangle \leq 0 \quad \forall x \in X .
$$

The following proposition is a special case of [19, Example 7.2], but we give its proof in order to make the paper self-contained. 
Proposition 15 (Continuity of $S$ ) If $S: E \rightrightarrows E^{*}$ is maximally monotone and singlevalued on $\operatorname{dom}(S)$, then $S$ is norm-to-weak* continuous.

Proof We first observe that $\operatorname{dom}(S)$ is open, since, for every $x \in \operatorname{dom}(S)$, the recession cone of $S x$ coincides with the normal cone to the closure of $\operatorname{dom}(S)$ at $x$ (see [20, Theorem 1] for the case when $E=\mathbb{R}^{n}$, and notice that the proof of this result extends to arbitrary Banach spaces in a straightforward way). Let $x \in \operatorname{dom}(S)$, and let $\left\{x_{n}\right\}_{n \in \mathbb{N}}$ be a sequence converging to $x$. Since $S$ is locally bounded at $x$, there exists a neighborhood $U$ of $x$ such that $S(U)$ is bounded. Without loss of generality, we assume that $\left\{x_{n}\right\}_{n \in \mathbb{N}}$ is contained in $U$; then the sequence $\left\{S x_{n}\right\}_{n \in \mathbb{N}}$ is bounded. Let $\left\{S x_{n_{k}}\right\}_{k \in \mathbb{N}}$ be any weakly* convergent subsequence, and denote its limit by $x^{*}$. Then, the sequence $\left\{\left(x_{n_{k}}, S x_{n_{k}}\right)\right\}_{k \in \mathbb{N}}$ converges to $\left(x, x^{*}\right)$; hence, since the graph of $S$ is closed (because of the maximal monotonicity of $S$ ), one has $x^{*}=S x$. Thus, in view of the Banach-Alaoglu Theorem, using the well-known fact that if all the convergent subsequences of a sequence in a compact space converge to a common limit then the whole sequence converges, we conclude that $\left\{S x_{n}\right\}_{n \in \mathbb{N}}$ converges to $S x$, thus proving continuity of $S$ at $x$.

Our next theorem will be a generalization of [15, Theorem 2]. To prove it, we will need the following lemma.

Lemma 16 Let $S$ and $h$ be as is Definition 2. If $X \subseteq E$, the operator $S$ is singlevalued on $\operatorname{dom}(S)$, and $h$ is norm $\times$ weak ${ }^{*}$ continuous, then, for any norm-to-norm continuous $f: X \rightarrow \operatorname{dom}(S)$, the function $X \times X \ni(x, y) \mapsto D_{h}(x, f(y)) \in \mathbb{R}$ is jointly strongly continuous.

Proof Let $\left\{\left(x_{n}, y_{n}\right)\right\}_{n \in \mathbb{N}}$ be a sequence in $X \times X$ strongly converging to $(x, y) \in$ $X \times X$. Similarly to the proof of Proposition 15, without loss of generality we may assume that the sequence $\left\{S f\left(y_{n}\right)\right\}_{n \in \mathbb{N}}$ is bounded. Then, since $\left\{x_{n}\right\}_{n \in \mathbb{N}}$ strongly converges to $x$ and $\left\{S f\left(y_{n}\right)\right\}_{n \in \mathbb{N}}$ weakly* converges to $S f(y)$, from the inequality $\left|\left\langle x_{n}, S f\left(y_{n}\right)\right\rangle-\langle x, S f(y)\rangle\right| \leq\left|\left\langle x_{n}-x, S f\left(y_{n}\right)\right\rangle\right|+\left|\left\langle x, S f\left(y_{n}\right)-S f(y)\right\rangle\right|$ we can easily deduce that $\left\{\left\langle x_{n}, S f\left(y_{n}\right)\right\rangle\right\}_{n \in \mathbb{N}}$ converges to $\langle x, S f(y)\rangle$. This, together with the norm-to-norm continuity of $f$, Proposition 15 , and the norm $\times$ weak* continuity of $h$, proves that $\left\{D_{h}\left(x_{n}, f\left(y_{n}\right)\right)\right\}_{n \in \mathbb{N}}$ converges to $D_{h}(x, f(y))$.

Theorem 17 (Fixed point theorem for the composition of $P_{X}^{h}$ with continuous mappings) Let $S$ and $h$ be as is Definition 2. If $X \subseteq E$ is nonempty, convex, and compact in the strong topology of $E$, the set $\operatorname{dom}(S)$ is convex, $S$ is single-valued on $\operatorname{dom}(S)$, and $h$ is norm $\times$ weak ${ }^{*}$ continuous, then, for any norm-to-norm continuous $f: X \rightarrow \operatorname{dom}(S)$, the mapping $P_{X}^{h} \circ f$ has a fixed point.

Proof The set

$$
A:=\left\{(x, y) \in X \times X: D_{h}(x, f(y)) \geq D_{h}(y, f(y))\right\}
$$

is closed by Lemma 16, and it obviously satisfies (i) of Lemma 1. It also satisfies ii), since $h$ is convex. Hence, by Lemma 1 , there exists $x_{0} \in X$ such that $X \times\left\{x_{0}\right\} \subseteq A$, 
that is,

$$
D_{h}\left(x_{0}, f\left(x_{0}\right)\right) \leq D_{h}\left(x, f\left(x_{0}\right)\right) \quad \forall x \in X
$$

equivalently,

$$
D_{h}\left(x_{0}, f\left(x_{0}\right)\right)=\min _{x \in X} D_{h}\left(x, f\left(x_{0}\right)\right)
$$

which means that $x_{0} \in P_{X}^{h}\left(f\left(x_{0}\right)\right)$; thus, $x_{0}$ is a fixed point of $P_{X}^{h} \circ f$.

To derive a (finite dimensional) result on classical Bregman distances from Theorem 17 , we need the following lemma.

Lemma 18 If $g: \mathbb{R}^{n} \rightarrow \mathbb{R} \cup\{+\infty\}$ is convex, continuous and supercoercive, then the function $h_{g}$ defined by (1) is continuous on $\mathbb{R}^{n} \times \mathbb{R}^{n}$.

Proof By [2, Theorem 3.4], the conjugate function $g^{*}$ is finite-valued; hence, since it is convex, it is continuous (see, e.g., [21, Corollary 10.1.1]). Consequently, as $h_{g}$ is a separable function with both terms being continuous, it is continuous, too.

Corollary 19 (Fixed point theorem for the composition of $P_{X}^{h_{g}}$ with continuous mappings) Let $X \subseteq \mathbb{R}^{n}$ be nonempty, convex and compact, and $g: \mathbb{R}^{n} \rightarrow \mathbb{R} \cup\{+\infty\}$ be proper, strictly convex, continuous, supercoercive, and differentiable on $\operatorname{dom}(g)$. Then, for any continuous mapping $f: X \rightarrow \operatorname{dom}(g)$, there exists a point $x_{0} \in X$ such that

$$
D^{g}\left(x_{0}, f\left(x_{0}\right)\right)=\min _{x \in X} D^{g}\left(x, f\left(x_{0}\right)\right) .
$$

Proof We apply Theorem 17 with $S:=\nabla g$, followed by Proposition 4. By Proposition 15, the mapping $\nabla g$ is continuous. We set $h:=h_{g}$, with $h_{g}$ defined by (1). By Lemma 18, the function $h_{g}$ is continuous. Therefore, given that $\operatorname{dom}(\nabla g)=$ $\operatorname{dom}(g)$ is convex, all the assumptions of Theorem 17 are satisfied; hence, we conclude that $P_{X}^{h_{g}} \circ f$ has a fixed point $x_{0}$. By Proposition 4, we obtain (3).

\section{Conclusions}

We have obtained convergence properties for Bregman-type distances associated with convex representations of maximally monotone operators. Such Bregman-type distances were introduced in [9] in such a way that when the maximally monotone operator is the (single-valued) subdifferential of a differentiable convex function $f$ and its convex representation $h$ is given by $h\left(x, x^{*}\right):=f(x)+f^{*}\left(x^{*}\right)$, the associated Bregman-type distance reduces to the classical Bregman distance induced by $f$. When we consider this particular situation, we recover some convergence results obtained earlier by Bauschke and Combettes [4]. 
Our Bregman-type distances induce, in a natural way, a notion of projection onto nonempty sets. Among other results for such projections, we prove a fixed point theorem for their compositions with continuous mappings, which generalizes a classical result of Fan [15] for ordinary projections in normed spaces.

Acknowledgements Juan Enrique Martínez-Legaz gratefully acknowledges financial support from the Spanish Ministry of Science, Innovation and Universities, through Grant PGC2018-097960-B-C21 and the Severo Ochoa Program for Centers of Excellence in R\&D (CEX2019-000915-S). He is affiliated with MOVE (Markets, Organizations and Votes in Economics). We are grateful to two anonymous reviewers, thanks to whose valuable remarks we have corrected some mistakes and improved the presentation.

Funding Open Access Funding provided by Universitat Autonoma de Barcelona.

Open Access This article is licensed under a Creative Commons Attribution 4.0 International License, which permits use, sharing, adaptation, distribution and reproduction in any medium or format, as long as you give appropriate credit to the original author(s) and the source, provide a link to the Creative Commons licence, and indicate if changes were made. The images or other third party material in this article are included in the article's Creative Commons licence, unless indicated otherwise in a credit line to the material. If material is not included in the article's Creative Commons licence and your intended use is not permitted by statutory regulation or exceeds the permitted use, you will need to obtain permission directly from the copyright holder. To view a copy of this licence, visit http://creativecommons.org/licenses/by/4.0/.

\section{References}

1. Alber, Y., Butnariu, D.: Convergence of Bregman projection methods for solving consistent convex feasibility problems in reflexive Banach spaces. J. Optim. Theory Appl. 92(1), 33-61 (1997)

2. Bauschke, H.H., Borwein, J.M., Combettes, P.L.: Essential smoothness, essential strict convexity, and Legendre functions in Banach spaces. Commun. Contemp. Math. 3(4), 615-647 (2001)

3. Bauschke, H.H., Chen, J., Wang, X.: A Bregman projection method for approximating fixed points of quasi-Bregman nonexpansive mappings. Appl. Anal. 94(1), 75-84 (2015)

4. Bauschke, H.H., Combettes, P.L.: Construction of best Bregman approximations in reflexive Banach spaces. Proc. Am. Math. Soc. 131(12), 3757-3766 (2003)

5. Bauschke, H.H., Combettes, P.L.: Iterating Bregman retraction. SIAM J. Optim. 13(4), 1159-1173 (2003)

6. Bregman, L.M.: The relaxation method of finding the common point of convex sets and its application to the solution of problems in convex programming. USSR Comput. Math. Math. Phys. 7(3), 200-217 (1967)

7. Burachik, R.S., Dao, M.N., Lindstrom, S.B.: The generalized Bregman distance. SIAM J. Optim. 31(1), 404-424 (2021)

8. Burachik, R.S., Fitzpatrick, S.: On a family of convex functions associated with subdifferentials. J. Nonlinear Convex Anal. 6(1), 165-171 (2005)

9. Burachik, R.S., Martínez-Legaz, J.E.: On Bregman-type distances for convex functions and maximally monotone operators. Set-Valued Var. Anal. 26(2), 369-384 (2018)

10. Burachik, R.S., Svaiter, B.F.: Maximal monotone operators, convex functions and a special family of enlargements. Set-Valued Anal. 10(4), 297-316 (2002)

11. Burachik, R.S., Svatier, B.F.: Operating enlargements of monotone operators: new connections with convex functions. Pac. J. Optim. 2(3), 425-445 (2006)

12. Censor, Y., Lent, A.: An iterative row-action method for interval convex programming. J. Optim. Theory Appl. 34(3), 321-358 (1981)

13. Chen, G., Teboulle, M.: Convergence analysis of a proximal-like minimization algorithm using Bregman functions. SIAM J. Optim. 3, 538-543 (1993)

14. Fan, K.: A generalization of Tychonoff's fixed point theorem. Math. Ann. 142, 305-310 (1960/61)

15. Fan, K.: Extensions of two fixed point theorems of F.E. Browder. Math. Z. 122, 234-240 (1969) 
16. Fitzpatrick, S.: Representing monotone operators by convex functions. Functional Analysis and Optimization, Workshop/Miniconference, Canberra/Australia 1988. Proc. Cent. Math. Anal. Aust. Natl. Univ., vol. 20, pp. 59-65 (1988)

17. Martínez-Legaz, J.-E., Svaiter, B.F.: Monotone operators representable by 1.s.c. convex functions. Set-Valued Anal. 13(1), 21-46 (2005)

18. Martínez-Legaz, J.-E., Tamadoni Jahromi, M., Naraghirad, E.: On farthest Bregman Voronoi cells. Optimization (2021). https://doi.org/10.1080/02331934.2021.1915313

19. Phelps, R.R.: Convex Functions, Monotone Operators and Differentiability. Springer, Berlin (1993)

20. Qi, L.: Complete closedness of maximal monotone operators. Math. Oper. Res. 8, 315-317 (1983)

21. Rockafellar, R.T.: Convex Analysis. Princeton University Press, Princeton (1970)

Publisher's Note Springer Nature remains neutral with regard to jurisdictional claims in published maps and institutional affiliations. 